\title{
Low Voltage Imaging of Quantum Materials Imaging the Surface Plasmon Polaritons in Chalcogenides
}

Cigdem Ozsoy-Keskinbora ${ }^{1}$, Kundan Chaudhary ${ }^{1}$, Michele Tamagnone ${ }^{1}$, Yunbo Ou ${ }^{2}$, Aravind Devarakonda ${ }^{3}$, Takehito Suzuki ${ }^{3}$, Joseph G Checkelsky ${ }^{3}$, Jagadeesh Moodera ${ }^{3}$, Federico Capasso ${ }^{1}$, David C. Bell ${ }^{1}$

1. Harvard John A. Paulson School of Engineering and Applied Sciences, Harvard University, Cambridge, USA.

2. Plasma Science and Fusion Center and Francis Bitter Magnet Laboratory, MIT, Boston, USA.

3. Department of Physics, MIT, Boston, USA.

Quantum Materials may act as conductors, insulators, semiconductors or even as superconductors. Especially combinations of different quantum materials are of high interest to explore new phenomena and to build the foundation for future electronic devices at the nanometer scale. The properties of quantum materials differ strongly from the properties of their three dimensional bulk state. Our research on quantum materials is widely spread, reaching from defect formation in graphene to the characterization of hybrid quantum materials. We use low voltage aberration corrected electron microscopy to investigate chemical various quantum materials. In particular, we will present imaging and analysis of vapor deposition (CVD) graphene with added copper and mercury defects; graphene based hybrid structures, and FeSn Topological Insulators.

The surface plasmon polaritons is a highly investigated field of research due to their high potential applications for sensors, information technologies, high-resolution imaging, to name a few. These collective delocalized electron oscillations are common at metal-dielectric interfaces. However, they also exist in highly doped semiconductors, conducting oxide systems or graphene, and in many other systems with high carrier mobility. This poses the question of whether such resonances can be observed at the insulator interfaces with these novel materials. Chalcogenides, the general name of sulfides, selenides, tellurides can show highly anisotropic crystal structures, that gives them exotic properties such as topological surface state, superconductivity or negative dielectric permittivity. It was already shown that $\mathrm{Bi}_{2} \mathrm{Se}_{3}$ supports Dirac plasmons, but the Dirac state is not the only reason for the existence of plasmon resonance in $\mathrm{Bi}_{2} \mathrm{Se}_{3}$. This material also has highly anisotropic dielectric properties allowing surface plasmon excitations, and it is not the only chalcogenide system that can support them. In this study we show electron energy loss spectroscopy and a finite-difference frequency-domain study for investigating $\mathrm{Bi}_{2} \mathrm{Se}_{3}$, and $\mathrm{BaNbS}$ (Figure 1.) with high spatial resolutions [1].

References:

[1] This work was supported by the STC Center for Integrated Quantum Materials, NSF Grant No. DMR-1231319. 
BaNbS cross section
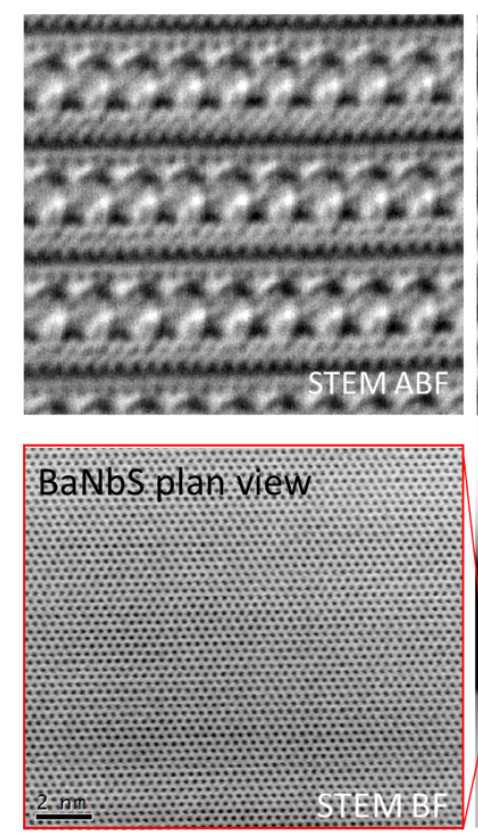

\section{- CIOM}
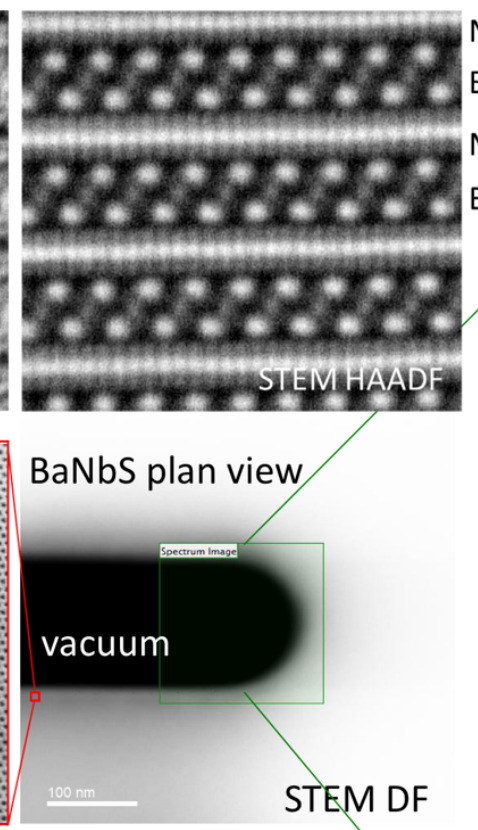

$\mathrm{Nb}-\mathrm{S}$

$\mathrm{Ba}-(\mathrm{Nb})-\mathrm{S}$

$\mathrm{Nb}-\mathrm{S}$

$\mathrm{Ba}-(\mathrm{Nb})-\mathrm{S}$

\section{Surface Plasmons in BaNbS}

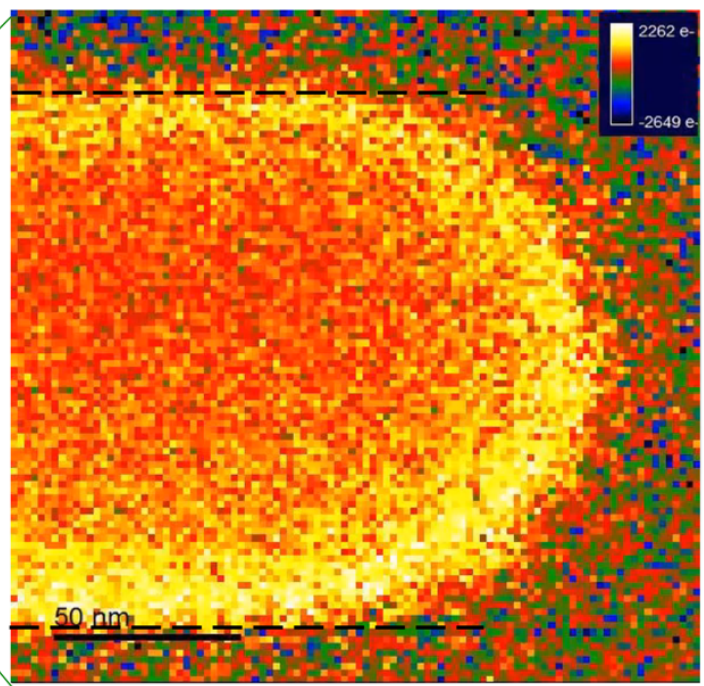

EELS SI 0.4 ev energy resolution $2.6-20.6 \mathrm{eV}$ range 76

Figure 1. Surface Plasmon Excitations in BaNbS. Top Left, HAABF, HAADF images on BaNbS cross section, Surface plasmon, energy filtered image of sample plan view. 